MSC $35 \mathrm{~K} 70,49 \mathrm{~K} 20$

DOI: $10.14529 / \mathrm{mmp} 200101$

\title{
OPTIMAL CONTROL IN LINEAR SOBOLEV TYPE MATHEMATICAL MODELS
}

\author{
A.A. Zamyshlyaeva ${ }^{1}$, N.A. Manakova ${ }^{1}$, O.N. Tsyplenkova ${ }^{1}$ \\ ${ }^{1}$ South Ural State University, Chelyabinsk, Russian Federation \\ E-mails: zamyshliaevaaa@susu.ru, manakovana@susu.ru, tcyplenkovaon@susu.ru
}

\begin{abstract}
The article presents a review of the work of the Chelyabinsk mathematical school on Sobolev type equations in studying the optimal control problems for linear Sobolev type models with initial Cauchy (Showalter-Sidorov) conditions or initial-final conditions. To identify the nonemptiness of the set of feasible solutions to the control problem we use the phase space method, which has already proved itself in solving Sobolev type equations. The method reduces the singular equation to a regular one defined on some subspace of the original space and applies the theory of degenerate (semi)groups of operators to the case of relatively bounded, sectorial and radial operators. Here mathematical models are reduced to initial (initial-final) problems for an abstract Sobolev type equation. Abstract results are applied to the study of control problems for the Barenblatt-Zheltov-Kochina mathematical model, which describes fluid filtration in a fractured-porous medium, the Hoff model on a graph simulating the dynamics of I-beam bulging in a construction, and the BoussinesqLöve model describing longitudinal vibrations in a thin elastic rod, taking into account inertia and under external load, or the propagation of waves in shallow water.

Keywords: Sobolev type equations; strong solutions; optimal control; phase space; Barenblatt-Zheltov-Kochina model; model of an I-beam bulging; Boussinesq-Löve model; Dzektzer model; Chen-Gurtin model.
\end{abstract}

\section{Introduction}

The research of the control problems for mathematical models based on Sobolev type equations is relevant due to the need to study important applied problems, in particular, in the theory of filtration, elasticity, biology and others. When studying mathematical models, it is important not only to understand the properties of the processes being studied, but also to be able to find the optimal regulation (external influence), with the help of which the state of the system takes the required value. The article describes methods and approaches developed in the framework of the scientific direction headed by G.A. Sviridyuk to study the optimal control problems for linear Sobolev type mathematical models with classical and non-classical initial (initial-final) conditions. A wide class of such mathematical models has been studied based on the theory of Sobolev type equations. Consider some of them.

The Barenblatt-Zheltov-Kochina model. Let $\Omega \subset \mathbb{R}^{n}$ be a bounded domain with a boundary $\partial \Omega$ of the class $C^{\infty}$. In the cylinder $\Omega \times \mathbb{R}_{+}$consider the Dirichlet boundary condition

$$
x(s, t)=0,(s, t) \in \partial \Omega \times \mathbb{R}
$$

for the Barenblatt-Zheltov-Kochina equation [1]

$$
(\lambda-\Delta) \dot{x}=\alpha \Delta x+u
$$

Вестник ЮУрГУ. Серия «Математическое моделирование

и программирование» (Вестник ЮУрГУ ММП). 2020. Т. 13, № 1. С. 5-27 
that simulates the process of fluid filtration in a fractured porous medium. Here $\alpha, \lambda \in \mathbb{R}$ characterize the properties of the medium; parameter $\alpha \in \mathbb{R}_{+}$, and parameter $\lambda$ can take negative values that do not contradict the physical meaning of the problem, the function $u=u(s, t)$ plays the role of an external exposure (i.e. characterizes the sources (drains) of the fluid). A mathematical model based on equation (2), supplemented by classical or non-classical initial (initial-final) conditions, was studied in various aspects in [2-4].

Mathematical model of an I-beam bugling. Let $\mathbf{G}=\mathbf{G}(\mathfrak{V} ; \mathfrak{E})$ be a finite connected oriented graph, where $\mathfrak{V}=\left\{V_{i}\right\}$ is a set of vertices, and $\mathfrak{E}=\left\{E_{i}\right\}$ is a set of edges. Each edge $E_{i}$ has the length $l_{i} \in \mathbb{R}_{+}$and the cross section area $d_{i} \in \mathbb{R}_{+}$. In each vertice $V_{i}, i=\overline{1, M}$, of the graph $\mathbf{G}$ consider the continuity conditions

$$
x_{j}(0, t)=x_{k}(0, t)=x_{m}\left(l_{m}, t\right)=x_{n}\left(l_{n}, t\right),
$$

where $E_{j}, E_{k} \in E^{\alpha}\left(V_{i}\right), E_{m}, E_{n} \in E^{\omega}\left(V_{i}\right)\left(E^{\alpha(\omega)}\left(V_{i}\right)\right.$ denotes the set of edges with the beginning (end) at the vertex $V_{i}$ ), and flow balance condition

$$
\sum_{E_{j} \in E^{\alpha}\left(V_{i}\right)} d_{j} x_{j s}(0, t)-\sum_{E_{k} \in E^{\omega}\left(V_{i}\right)} d_{k} x_{k s}\left(l_{k}, t\right)=0
$$

for Hoff equations [5]

$$
\lambda x_{j t}+x_{j t s s}=\alpha_{j} x_{j}+u_{j} .
$$

Conditions (3), (4) and equation (5) form the Hoff mathematical model on a graph that describes the dynamics of deformation of an I-beam construction under constant load $\lambda \in \mathbb{R}_{+}$. Parameters $\alpha_{j} \in \mathbb{R}$ characterize beam material properties; the free term $u_{j}=u_{j}(s, t)$ corresponds to the external (lateral) load on the $j$-th edge of the graph. Equations (5) on graphs were first studied in [6]. The Hoff mathematical model in the domain or on the graph, supplemented by classical or non-classical initial (initial-final) conditions in various aspects was studied in [7-9].

The Boussinesq-Löve model. Let $\Omega \subset \mathbb{R}^{n}$ be a bounded domain with a boundary $\partial \Omega$ of the class $C^{\infty}$. In the cylinder $\Omega \times \mathbb{R}$ consider the Boussinesq-Löve equation [10]

$$
(\lambda-\Delta) x_{t t}=\beta\left(\Delta-\lambda^{\prime}\right) x+u
$$

with boundary condition (1). Model (1), (6) describes the propagation of waves in shallow water. Parameters $\beta, \lambda, \lambda^{\prime}$ relate to depth, gravitational constant and Bond number. The function $x(t, s)$ determines the wave height at time $t$ at the point $s, u(t, s)$ is a control that defines external forces. The Boussinesq-Löve mathematical model, supplemented by classical or non-classical initial (initial-final) conditions, was studied in various aspects in $[10,11]$.

The Dzektzer model. Let $\Omega \subset \mathbb{R}^{n}$ be a bounded domain with a boundary $\partial \Omega$ of the class $C^{\infty}$. In the cylinder $\Omega \times \mathbb{R}_{+}$consider the problem

$$
x(s, t)=\Delta x(s, t)=0,(s, t) \in \partial \Omega \times \mathbb{R}_{+}
$$

for a non-classical partial differential equation [12]

$$
(\lambda-\Delta) \dot{x}=\alpha \Delta x-\beta \Delta^{2} x+u .
$$


The mathematical model (7), (8) describes the evolution of the free surface of the filtered fluid. Here $\alpha, \beta \in \mathbb{R}_{+}, \lambda \in \mathbb{R}$ are the parameters characterizing the fluid, the free term $u=u(s, t)$ characterizes the sources (drains) of the fluid. Mathematical model based on equation (8), supplemented by classical or non-classical initial (initial-final) conditions in various aspects was studied in $[13,14]$.

The Chen-Gurtin model with complex coefficients. Let $\Omega \subset \mathbb{R}^{n}$ be a bounded domain with a boundary $\partial \Omega$ of class $C^{\infty}$. In the cylinder $\Omega \times \mathbb{R}_{+}$consider the boundary value problem (7) for a non-classical partial differential equation [15]

$$
(\lambda-\Delta) x_{t}=\nu \Delta x-i d \Delta^{2} x+u \text {. }
$$

Here the coefficients $\nu, \lambda, d \in \mathbb{R}$ characterize parameters of the system. The required complex-valued function $x(s, t)$ describes the dynamics of the process, and the complexvalued function $u(s, t)$ describes an external effect on the system. In the special case of $d=0$ equation (9) describes the process of heat conduction with "two temperatures" [15], as well as the dynamics of fluid pressure in a cracks-porous medium [1]. A mathematical model based on equation (9), supplemented by classical non-classical initial (initial-final) conditions in various aspects was studied in $[16,17]$.

The mathematical models under consideration belong to a wide class of Sobolev type models (i.e., models based on Sobolev type equations). Sobolev type equations currently constitute a significant part of the non-classical equations of mathematical physics. Initially, such equations arose in the works of A. Poincare, C. Rossby, J. Boussinesq and other mechanics in the late XIX - early XX centuries. However, a systematic study of such equations began in the middle of the last century with the work of S.L. Sobolev. A detailed history of this issue can be found in the monograph [18]. Note that various terms are used in literature to denote such equations [18-20]. The term "Sobolev type equations" $[3,21]$ first appeared in the works of R.E. Showalter [22]. We adhere to this term, considering the rest as synonyms.

The mentioned mathematical models with one or another initial (initial-final) conditions in suitable Banach spaces can be reduced to the corresponding problems for a linear Sobolev type equation

$$
A x^{(n)}=B x+y+C u,
$$

where operators $A \in \mathcal{L}(\mathfrak{X} ; \mathfrak{Y}), B \in \mathcal{C l}(\mathfrak{X} ; \mathfrak{Y}), C \in \mathcal{L}(\mathfrak{U} ; \mathfrak{Y})$, functions $u: \mathfrak{I} \rightarrow \mathfrak{U}$, $y: \mathfrak{I} \rightarrow \mathfrak{Y}(\mathfrak{I} \subset \mathbb{R})$, and $\mathfrak{X}, \mathfrak{Y}, \mathfrak{U}$ are Hilbert spaces. To select the unique process under study, the mathematical models under consideration and their abstract interpretation (10) are supplemented by one of the following conditions:

- the Cauchy condition [3]

$$
x^{(m)}(0)=x_{m}, m=0, \ldots n-1,
$$

- the Showalter-Sidorov condition $[23,24]$

$$
P\left(x^{(m)}(0)-x_{m}\right)=0, m=0, \ldots, n-1,
$$

- the initial-final condition $[4,25]$

$$
P_{\text {in }}\left(x^{(m)}(0)-x_{m}^{0}\right)=0, P_{\text {fin }}\left(x^{(m)}(\tau)-x_{m}^{\tau}\right)=0, m=0, \ldots, n-1,
$$


where $P, P_{\text {in }}, P_{\text {fin }}$ are some spectral projectors in the space $\mathfrak{X}$, which will be defined later. Condition (13) differs from the initial conditions in that one projection of the solution is specified at the initial moment of time, and the other is set at the final moment of the considered time interval. The initial-final condition is a generalization of the ShowalterSidorov condition, which in turn is a generalization of the classical Cauchy condition. As it is well known (see, for example, [23]), the Cauchy problem for the Sobolev type equation (10) (in case ker $A \neq\{0\}$ ) is not solvable for arbitrary initial values $x_{m}, m=\overline{0, n-1}$. To overcome this difficulty, G.A. Sviridyuk proposed the phase space method. The foundations of this concept were laid down in [20], then the concept was developed in [3] and many other works. Another approach to overcome the difficulties associated with non-existence of the solution to (10), (11) is to consider the initial Showalter-Sidorov condition (12) and a more general initial-final condition (13) instead of the initial Cauchy condition (11). We are interested in solving the optimal control problem, which consists in finding a pair $(\hat{x}, \hat{u})$, for which the relation

$$
J(\hat{x}, \hat{u})=\min _{(x, u) \in \mathfrak{X} \times \mathfrak{U}_{a d}} J(x, u)
$$

holds. Here the pairs $(x, u)$ satisfy the Cauchy problem $(10),(11)$ or the Showalter-Sidorov problem (10), (12), or the initial-final problem (10), (13) and $J(x, u)$ is some specially constructed quality functional, $\mathfrak{U}_{a d}$ is some closed and convex set in the control space $\mathfrak{U}$.

The article provides an overview of the results developed in the framework of the direction headed by G.A. Sviridyuk on the optimal control of the solutions to the initialfinal problem and, in particular, the Showalter-Sidorov and Cauchy problems for linear Sobolev type equations. The first who began to study the controllability problems and the optimal control problem for linear Sobolev type equations with the Cauchy condition were G.A. Sviridyuk and A.A. Efremov $[2,13,26]$. In these papers, the optimal control problem with a quadratic quality functional was studied in case $n=1$ with $(A, p)$-bounded or $(A, p)$ sectorial operator $B$ and the Cauchy condition, the necessary and sufficient conditions for the existence and uniqueness of a solution were obtained. G.A. Sviridyuk suggested moving from considering the classical solution $x \in C^{1}(\mathfrak{J} ; \mathfrak{X})$ of $(10)$, (11) to the strong solution $x \in H^{p+1}(\mathfrak{X})$ of this problem, which allowed to set the optimal control problem (10), (11), (14) and to use the technique of Hilbert spaces for its research. These studies formed the basis of a number of works by G.A. Sviridyuk's disciples and followers on the study of optimal control problems for linear Sobolev type equations based on the theory of degenerate resolving (semi)groups of operators [3]. Since [2,3,13], when considering the classical Cauchy condition, due to the degeneracy of the equation, it was necessary to reconcile the initial data with the control action, then G.A. Sviridyuk suggested an idea to use more general initial Showalter-Sidorov condition (initial-final condition), which made it possible to remove the restriction on the set of optimal controls in the subsequent works of his disciples and followers and opened the way to a whole class of problems on this subject $[8,27]$. In [10] the necessary and sufficient conditions for the existence and uniqueness of the solution of optimal control problems for high-order Sobolev type equations with an initial-final condition were obtained. The ideas and methods developed by G.A. Sviridyuk and A.A. Efremov on controllability of linear abstract Sobolev type equation opened the way to the study of more general controllability problems [28].

The article consists of introduction, 6 sections and conclusion. The first Section gives the main points of the theory of relatively bounded operators, the complete proofs of 
which can be found in [3]. It contains theorems on existence and uniqueness of classical solution of optimal control problems for an abstract Sobolev type equations, as well as on existence and uniqueness of strong solution to the initial (initial-final) problem for such equations. In the second Section, the abstract results are applied to specific Sobolev type models, namely, the Barenblatt-Zheltovaya-Kochina model, the Hoff model on the graph, and the Boussinesq-Löve model. The third Section contains the results of the theory regarding relatively sectorial operators for the first-order Sobolev type equation. In the fourth Section, the Dzektzer model and the pressure evolution model on the graph are reduced to an abstract Sobolev type equation with initial (initial-final) conditions, and then the abstract results are applied to the study of optimal control problem for them. The fifth Section contains the main results of the theory with relatively radial operators. The optimal control in the Chen-Gurtin model based on the Sobolev type equation with a relatively radial operator is studied in the sixth Section.

\section{Relatively $p$-Bounded Operators. Strong Solutions. Optimal Control}

In this section definitions and results of the theory of relatively bounded operators are given $[3,29]$. Let $\mathfrak{X}, \mathfrak{Y}$ and $\mathfrak{U}$ be Banach spaces. Operators $A, B \in \mathcal{L}(\mathfrak{X} ; \mathfrak{Y})$, operator $C \in \mathcal{L}(\mathfrak{U} ; \mathfrak{Y})$. The set

$$
\rho^{A}(B)=\left\{\mu \in \mathbb{C}:(\mu A-B)^{-1} \in \mathcal{L}(\mathfrak{Y} ; \mathfrak{X})\right\}
$$

is called a resolvent set of operator $B$ with respect to operator $A$ (an $A$-resolvent set of the operator $B$ ). The set $\mathbb{C} \backslash \rho^{A}(B)=\sigma^{A}(B)$ is called a spectrum of operator $B$ with respect to operator $A$ (an $A$-spectrum of operator $B$ ). The operator function

$$
(\mu A-B)^{-1}, \quad R_{\mu}^{A}=(\mu A-B)^{-1} A, \quad L_{\mu}^{A}=A(\mu A-B)^{-1}
$$

of a complex variable with domain $\rho^{A}(B)$ is called a resolvent, a right resolvent, a left resolvent of operator $B$ with respect to operator $A$ (an $A$-resolvent, a right $A$-resolvent, a left A-resolvent of operator $B$ ).

Definition 1. The operator $B$ is called polynomially bounded with respect to operator $A$ (or simply $(\mathrm{A}, \sigma)$-bounded), if

$$
\exists a>0 \forall \mu \in \mathbb{C}:(|\mu|>a) \Rightarrow\left(\mu \in \rho^{A}(B)\right) .
$$

Lemma 1. [29] If the operator $B$ is $(A, \sigma)$-bounded, then the following operators

$$
P=\frac{1}{2 \pi i} \int_{\Gamma} R_{\mu^{n}}^{A}(B) \mu^{n-1} A d \mu, \quad Q=\frac{1}{2 \pi i} \int_{\Gamma} \mu^{n-1} A R_{\mu^{n}}^{A}(B) d \mu
$$

are projectors, moreover $P: \mathfrak{X} \rightarrow \mathfrak{X}$ and $Q: \mathfrak{Y} \rightarrow \mathfrak{Y}$. Here $\Gamma=\left\{\lambda \in \mathbb{C}:\left|\lambda^{n}\right|=r>a\right\}$.

Set $\mathfrak{X}^{0}=\operatorname{ker} P, \mathfrak{Y}^{0}=\operatorname{ker} Q, \mathfrak{X}^{1}=\operatorname{im} P, \mathfrak{Y}^{1}=\operatorname{im} Q$. By $A_{k}$ denote the restriction of the operator $A$, and by $B_{k}$ denote the restriction of the operator $B$ to $\mathfrak{X}^{k}, k=0,1$. The theorem of operator actions' splitting is true. 
Theorem 1. [3, Ch. 4] Let the operator $B$ be $(A, \sigma)$-bounded. Then

(i) operators $A_{k}, B_{k}: \mathfrak{X}^{k} \rightarrow \mathfrak{Y}^{k}, k=0,1$;

(ii) there exists an operator $B_{0}^{-1} \in \mathcal{L}\left(\mathfrak{Y}^{0}, \mathfrak{X}^{0}\right)$;

(iii) there exists an operator $A_{1}^{-1} \in \mathcal{L}\left(\mathfrak{Y}^{1}, \mathfrak{X}^{1}\right)$;

(iv) operator $B_{1} \in \mathcal{L}\left(\mathfrak{X}^{1}, \mathfrak{Y}^{1}\right)$.

Let us construct the operators $H=B_{0}^{-1} A_{0} \in \mathcal{L}\left(\mathfrak{X}^{0}\right)$ and $S=A_{1}^{-1} B_{1} \in \mathcal{L}\left(\mathfrak{X}^{1}\right)$. Then

$$
(\mu A-B)^{-1}=\left(-\sum_{k=0}^{\infty} \mu^{k} H^{k}\right) B_{0}^{-1}(\mathbb{I}-Q)+\sum_{k=1}^{\infty} \mu^{-k} S^{k-1} A_{1}^{-1} Q .
$$

Definition 2. The point $\infty$ is called:

(i) a removable singular point of the A-resolvent of the operator $B$, if $H \equiv \mathbb{O}$;

(ii) a pole of order $p$ of the A-resolvent of the operator $B$, if $H^{p} \neq \mathbb{O}, H^{p+1} \equiv \mathbb{O}$, $p \in \mathbb{N}$

(iii) an essentially singular point of the A-resolvent of the operator $B$, if $H^{q} \neq \mathbb{O}$, $\forall q \in \mathbb{N}$.

Definition 3. $(A, \sigma)$-bounded operator $B$ is called $(A, p)$-bounded for some $p \in\{0\} \cup \mathbb{N}$, if the point $\infty$ is a pole of order $p \in\{0\} \cup \mathbb{N}$ of the A-resolvent of operator $B$.

Definition 4. The vector-function $x \in C^{n}(\mathbb{R} ; \mathfrak{X}), n \in \mathbb{N}$, satisfying (10) is called a classical solution of this equation.

Consider linear homogeneous $(y=u=0)$ Sobolev type equation (10).

Definition 5. The operator-function $V^{\bullet} \in C^{\infty}(\mathbb{R} ; \mathcal{L}(\mathfrak{X}))$ is called a propagator of homogeneous equation (10), if for arbitrary $v \in \mathfrak{X}$ the vector-function $x(t)=V^{t} v$ is the solution of this equation.

Theorem 2. [29] If the operator $B$ is $(A, \sigma)$-bounded, then formulas

$$
X_{m}^{t}=\frac{1}{2 \pi i} \int_{\Gamma} \mu^{n-m-1}\left(\mu^{n} A-B\right)^{-1} A e^{\mu t} d \mu, m=\overline{0, n-1},
$$

define propagators of a homogeneous equation (10) for $t \in \mathbb{R}$.

Consider the inhomogeneous equation

$$
A x^{(n)}=B x+y
$$

and sets

$$
\mathcal{M}_{y}^{m}=\left\{x \in \mathfrak{X}:(\mathbb{I}-P) x=-\sum_{q=0}^{p} H^{q} B_{0}^{-1} \frac{d^{n q+m}}{d t^{n q+m}}(\mathbb{I}-Q) y(0)\right\}, m=\overline{0, n-1} .
$$

The results on existence and uniqueness of a classical solution to (11), (16) were obtained in $[10,29]$.

Theorem 3. [29] Let the operator $B$ be $(A, p)$-bounded for some $p \in\{0\} \cup \mathbb{N}$, the function $y: \mathfrak{I} \rightarrow \mathfrak{Y}(\mathfrak{I} \subset \mathbb{R})$ be such that $y^{0}=(\mathbb{I}-Q) y \in C^{n p+n}\left(\mathfrak{I} ; \mathfrak{Y}^{0}\right)$ and $y^{1}=Q y \in C\left(\mathfrak{I} ; \mathfrak{Y}^{1}\right)$. 
Let the initial values $x_{m} \in \mathcal{M}_{y}^{m}, m=\overline{0, n-1}$. Then there exists a unique classical solution to the Cauchy problem (11), (16) given by

$$
x(t)=-\sum_{q=0}^{p} H^{q} B_{0}^{-1}(\mathbb{I}-Q) y^{(q n)}(t)+\sum_{m=0}^{n-1} X_{m}^{t} x_{m}+\int_{0}^{t} X_{n-1}^{t-s} A_{1}^{-1} Q y(s) d s, t \in \mathfrak{I} .
$$

There were obtained results [10] on the existence and uniqueness of a classical solution to problem (12), (16). Note that when using the Showalter-Sidorov condition, the assumptions of Theorem 3 can be weakened without requiring initial data being agreed with the right side of equation (16).

Theorem 4. [29] Let the operator $B$ be $(A, p)$-bounded for some $p \in\{0\} \cup \mathbb{N}$, the function $y: \mathfrak{I} \rightarrow \mathfrak{Y}$ be such that $y^{0}=(\mathbb{I}-Q) y \in C^{n p+n}\left(\mathfrak{I} ; \mathfrak{Y}^{0}\right)$ and $y^{1}=Q y \in C\left(\mathfrak{I} ; \mathfrak{Y}^{1}\right)$. Then for arbitrary $x_{m} \in \mathfrak{X}, m=\overline{0, n-1}$, there exists a unique classical solution to ShowalterSidorov problem (12), (16) given by (18).

Proceed to consider a more general than the Showalter-Sidorov initial-final condition. Introduce an additional condition

$$
\sigma^{A}(B)=\sigma_{0}^{A}(B) \cup \sigma_{1}^{A}(B), \sigma_{k}^{A}(B) \neq \emptyset, k=\overline{0,1} ;
$$

and contour $\gamma_{0}$ is the boundary of domain $D \subset \mathbb{C}$ such that

$$
D \cap \sigma_{0}^{A}(B)=\sigma_{0}^{A}(\vec{B}), \bar{D} \cap \sigma_{1}^{A}(B)=\emptyset .
$$

Then there exists an operator

$$
P_{\text {fin }}=\frac{1}{2 \pi i} \int_{\gamma_{0}} R_{\mu^{n}}^{A}(B) \mu^{n-1} A d \mu \in \mathcal{L}(\mathfrak{X}) .
$$

Lemma 2. [29] Let the operator $B$ be $(A, p)$-bounded for some $p \in\{0\} \cup \mathbb{N}$, let condition $(B)$ be satisfied. Then $P_{\text {fin }}$ is a projector, and $P_{\text {fin }} P=P P_{\text {fin }}=P_{\text {fin }}$.

Construct an operator $P_{\text {in }}=P-P_{\text {fin }} \in \mathcal{L}(\mathfrak{X})$. By Lemma 2 operator $P_{\text {in }}$ is a projector. Consider operators

$$
X_{\text {fin }}^{m}(t)=\frac{1}{2 \pi i} \int_{\gamma_{0}} R_{\mu}^{A}(\vec{B})\left(\mu^{n-m-1} A-B\right) e^{\mu t} d \mu .
$$

Note that $X_{f i n}^{k}$ is a propagator of homogeneous $(y=0)$ equation (16). Introduce a family of operators

$$
X_{i n}^{k}(t)=X_{k}^{t}-X_{\text {fin }}^{k}(t), \quad k=\overline{0, n-1} .
$$

Theorem 5. [29] Let the operator $B$ be $(A, p)$-bounded for some $p \in\{0\} \cup \mathbb{N}$. Let the vector function $y: \mathfrak{I} \rightarrow \mathfrak{Y}$ be such that $y^{0}=(\mathbb{I}-Q) y \in C^{n p+n}\left(\mathfrak{I} ; \mathfrak{Y}^{0}\right)$ and $y^{1}=Q y \in C\left(\mathfrak{I} ; \mathfrak{Y}^{1}\right)$. Then for arbitrary $x_{m}^{0}, x_{m}^{\tau} \in \mathfrak{X}, m=\overline{0, n-1}$, there exists a unique classical solution to problem (13), (16), for $t \in \mathfrak{I}$ given by

$$
\begin{aligned}
x(t)=- & \sum_{q=0}^{p} H^{q}\left(B_{0}\right)^{-1}(\mathbb{I}-Q) y^{(n q)}(t)+\sum_{m=0}^{n-1} X_{i n}^{m}(t) P_{i n} x_{m}^{0}+\sum_{m=0}^{n-1} X_{f i n}^{m}(t) P_{f i n} x_{m}^{\tau}+ \\
& +\int_{0}^{t} X_{i n}^{n-1}(t-s) A_{1}^{-1} P_{i n} y(s) d s-\int_{t}^{\tau} X_{f i n}^{n-1}(t-s) A_{1}^{-1} P_{f i n} y(s) d s .
\end{aligned}
$$


Thus, a general theory that allows one to find classical solutions for (16) with initial (initial-final) conditions is constructed. Now turn to the study of control problems. It should be noted that in such problems the technique of Hilbert spaces is traditionally used, which requires consideration of other types of solutions. Further we consider $\mathfrak{X}, \mathfrak{Y}$ and $\mathfrak{U}$ being Hilbert spaces. Consider space $H^{k}(\mathfrak{Y})=\left\{v \in L_{2}(0, \tau ; \mathfrak{Y}): v^{(k)} \in L_{2}(0, \tau ; \mathfrak{Y})\right\}$. The space $H^{k}(\mathfrak{Y})$ is Hilbert with inner product

$$
[v, w]=\sum_{q=0}^{k} \int_{0}^{\tau}\left\langle v^{(q)}, w^{(q)}\right\rangle_{\mathfrak{Y}} d t
$$

Definition 6. The vector-function $x \in H^{n}(\mathfrak{X})=\left\{x \in L_{2}(\mathfrak{I} ; \mathfrak{X}): x^{(n)} \in L_{2}(\mathfrak{I} ; \mathfrak{X})\right\}$ is called a strong solution to (16), if it turns the equation to an identity almost everywhere on interval $\mathfrak{I}$. A strong solution $x=x(t)$ of (16) is called a strong solution of the Cauchy problem, if condition (11) holds; a strong solution of the Showalter-Sidorov problem, if condition (12) holds; a strong solution of the initial-final problem, if condition (13) holds.

Note that classical solution (18) is also a strong solution to equation (16) by virtue of the continuity of the embedding $H^{n}(\mathfrak{X}) \hookrightarrow C^{n-1}(\mathfrak{I} ; \mathfrak{X})$.

Theorem 6. [30] Let the operator $B$ be $(A, p)$-bounded for some $p \in\{0\} \cup \mathbb{N}$. Then for arbitrary $y \in H^{n p+n}(\mathfrak{Y}), x_{m} \in \mathcal{M}_{y}^{m}, m=\overline{0, n-1}$, there exists a unique strong solution to the Cauchy problem (11), (16).

Theorem 7. [31] Let the operator $B$ be $(A, p)$-bounded for some $p \in\{0\} \cup \mathbb{N}$. Then for arbitrary $x_{m}^{0}, x_{m}^{\tau} \in \mathfrak{X}, m=\overline{0, n-1}$, and $y \in H^{n p+n}(\mathfrak{Y})$ there exists a unique strong solution to the initial-final problem (13), (16).

Note that in the case of $\left(\sigma_{i n}^{A}=\sigma^{A}(B), \sigma_{\text {fin }}^{A}=\emptyset\right)$ the Showalter-Sidorov problem can be considered as a special case of the initial-final problem. The following result follows from Theorem 7 .

Corollary 1. Let the operator $B$ be $(A, p)$-bounded for some $p \in\{0\} \cup \mathbb{N}$. Then for arbitrary $x_{m} \in \mathfrak{X}, m=\overline{0, n-1}$, and $y \in H^{n p+n}(\mathfrak{Y})$ there exists a unique strong solution to the Showalter-Sidorov problem (12), (16).

Definition 7. The pair $(\hat{x}, \hat{u})$ is called a solution to the optimal control problem (10), (11), (14) if relation (14) is satisfied and all pairs $(x, u) \in H^{n p+p}(\mathfrak{X}) \times \mathfrak{U}_{a d}$ are solutions of problem (10), (11). A vector function $\hat{u} \in \mathfrak{U}_{a d}$ is called an optimal control of solutions to (10), (11).

Consider the penalty functional

$$
J(x, u)=\mu \sum_{q=0}^{n} \int_{0}^{\tau}\left\|x^{(q)}-\tilde{x}^{(q)}\right\|^{2} d t+\nu \sum_{q=0}^{n p+n} \int_{0}^{\tau}\left\langle N_{q} u^{(q)}, u^{(q)}\right\rangle_{\mathfrak{U}} d t .
$$

Here $\mu, \nu>0, \mu+\nu=1, N_{q} \in \mathcal{L}(\mathfrak{U}), q=0,1, \ldots, n p+n$, are self-adjoint positively defined operators, and $\tilde{x}(t)$ is the target state of the system. 
Remark 1. By Theorem 7 on the existence of a unique solution for all $y \in H^{n p+n}(\mathfrak{Y})$ and $u \in \mathfrak{U}_{a d}$ the solution $x=x(u)$. Therefore, the quality functional depends only on $u$ : $J(x, u)=J(u)$. Thus, the set of feasible solutions to problem (10), (11), (14) is not empty.

When using the Cauchy condition, we pass to consideration of the subspace of controls

$$
\stackrel{\circ}{H}^{n p+n}(\mathfrak{U})=\left\{u \in L_{2}(0, \tau ; \mathfrak{U}): u^{(n p+n)} \in L_{2}(0, \tau ; \mathfrak{U}), u^{(q)}(0)=0, q=\overline{0, n p+n-1}\right\} .
$$

In the space $\stackrel{\circ}{H}^{n p+n}(\mathfrak{U})$ we single out a closed convex subset $\mathfrak{U}_{a d} \subset \stackrel{\circ}{H}^{n p+n}(\mathfrak{U})$, which will be called the set of admissible controls. Results on existence and uniqueness of optimal control of solutions to the Cauchy problem (10), (11) were obtained in [30].

Theorem 8. [30] If the operator $B$ is $(A, p)$-bounded for some $p \in\{0\} \cup \mathbb{N}$, then for arbitrary $y \in H^{n p+n}(\mathfrak{Y})$ and $x_{m} \in \mathcal{M}_{y}^{m}, m=\overline{0, n-1}$, there exists a unique optimal control of solutions to problem (10), (11).

Introduce the control space $H^{n p+n}(\mathfrak{U})$. Single out a closed convex subset $\mathfrak{U}_{a d} \subset$ $H^{n p+n}(\mathfrak{U})$. Results on existence and uniqueness of optimal control of solutions to initialfinal problem (10), (13) were obtained in [31].

Theorem 9. [31] If the operator $B$ is $(A, p)$-bounded for some $p \in\{0\} \cup \mathbb{N}$, then for arbitrary $y \in H^{n p+n}(\mathfrak{Y})$ and $x_{m}^{0}, x_{m}^{\tau} \in \mathfrak{X}, m=\overline{0, n-1}$, there exists a unique optimal control of solutions to the initial-final problem (10), (13).

The following result follows from Theorem 9.

Corollary 2. If the operator $B$ is $(A, p)$-bounded for some $p \in\{0\} \cup \mathbb{N}$, then for arbitrary $x_{m} \in \mathfrak{X}, m=\overline{0, n-1}$, and $y \in H^{n p+n}(\mathfrak{Y})$ there exists a unique optimal control to solutions of the Showalter-Sidorov problem (10), (12).

\section{Optimal Control in Sobolev Type Models with Relatively $p$-Bounded Operators}

Optimal control in the Barenblatt-Zheltov-Kochina model. Consider the optimal control problem (14) for mathematical model (1), (2) with the initial Cauchy condition (11). Reduce this problem to equation (10) with $n=1$. For this purpose put

$$
\mathfrak{X}=H^{2}(\Omega) \cap \stackrel{o}{H^{1}}(\Omega), \quad \mathfrak{Y}=L_{2}(\Omega), \quad \mathfrak{U}=L_{2}(\Omega),
$$

and by formulas

$$
A=\lambda \mathbb{I}-\Delta, \quad B=\alpha \Delta, \quad C=\mathbb{I},
$$

define the operators $A, B \in \mathcal{L}(\mathfrak{X}, \mathfrak{Y})$. Denote by $\left\{\varphi_{k}\right\}$ the set of eigenfunctions of the Dirichlet problem for the Laplace operator $\Delta$, numbered in ascending order of eigenvalues $\left\{\lambda_{k}\right\}$ taking into account their multiplicity. Consider the $A$-resolvent of operator $B$ in the form

$$
(\mu A-B)^{-1}=\sum_{k=1}^{\infty} \frac{\left\langle\cdot, \varphi_{k}\right\rangle \varphi_{k}}{\mu\left(\lambda-\lambda_{k}\right)-\alpha \lambda_{k}},
$$


where $\langle\cdot, \cdot\rangle$ is an inner product in $L_{2}(\Omega)$. Hence, the $A$-spectrum of operator $B$ is given by

$$
\sigma^{A}(B)=\left\{\mu_{k}=\frac{\alpha \lambda_{k}}{\lambda-\lambda_{k}}: \lambda \neq \lambda_{k}\right\} .
$$

Lemma 3. [3] The operator $B$ defined by (22) is $(A, 0)$-bounded for any $\lambda \in \mathbb{R}$. form

Let the function $y \in H^{1}(\mathfrak{Y})$. Then the set $\mathcal{M}_{y}$ (given by $(18)$ for $n=1$ ) takes the

$$
\mathcal{M}_{y}=\left\{x \in \mathfrak{X}:-\lambda \alpha\left\langle x, \varphi_{k}\right\rangle=\left\langle y(0), \varphi_{k}\right\rangle, \lambda_{k}=\lambda\right\}
$$

and the control space

$$
\stackrel{o}{H^{1}}(\mathfrak{U})=\left\{u \in L_{2}(0, \tau ; \mathfrak{U}): \dot{u} \in L_{2}(0, \tau ; \mathfrak{U}), u(0)=0\right\} .
$$

Choose a closed convex subset $\mathfrak{U}_{a d}$ in it.

Theorem 10. [2] For any $y \in H^{1}(\mathfrak{Y})$ and $x_{0} \in \mathcal{M}_{y}$ there exists a unique solution $(\hat{x}, \hat{u}) \in$ $H^{1}(\mathfrak{X}) \times \mathfrak{U}_{\text {ad }}$ to the optimal control problem (14) for mathematical model (1), (2) with the initial Cauchy condition (11), that minimizes functional (20).

Remark 2. The vector function $\hat{x} \in H^{1}(\mathfrak{X})$ defines the desired distribution of pressure in $\Omega$, and the control $\hat{u}$ means the effect on pressure by increasing (decreasing) the influence of liquid sources (drains) in $\Omega$.

Optimal control in the Hoff model on a graph. Consider the optimal control problem for mathematical model (3) - (5) with initial-final condition (13) in the case of $n=1$. Reduce the problem to equation (10) with $n=1$. Introduce the Hilbert space $L_{2}(\mathbf{G})=$ $\left\{u=\left(u_{1}, u_{2}, \ldots, u_{j}, \ldots\right): u_{j} \in L_{2}\left(0, l_{j}\right)\right\}$ with inner product

$$
\langle u, v\rangle=\sum_{E_{j} \in \mathfrak{E}} d_{j} \int_{0}^{l_{j}} u_{j} v_{j} d s,
$$

and Banach space $\mathfrak{X}=\left\{x=\left(x_{1}, x_{2}, \ldots, x_{j}, \ldots\right): x_{j} \in H^{1}\left(0, l_{j}\right)\right.$ and (3) holds $\}$ with norm

$$
\|x\|_{\mathfrak{X}}^{2}=\sum_{E_{j} \in \mathfrak{E}} d_{j} \int_{0}^{l_{j}}\left(x_{j s}^{2}+x_{j}^{2}\right) d s .
$$

Denote by $\mathfrak{Y}$ the conjugate space to $\mathfrak{X}$ with respect to the duality $\langle\cdot, \cdot\rangle$ and by formula

$$
\langle L x, v\rangle=-\sum_{E_{j} \in \mathfrak{E}} d_{j} \int_{0}^{l_{j}} x_{j s} v_{j s} d s, \quad x, v \in \mathfrak{X}
$$

define the operator $L \in \mathcal{L}(\mathfrak{X} ; \mathfrak{Y})$. It was shown [32] that $\sigma(L)$ is negative, discrete, with finite multiplicity, and condenses only to $-\infty$. Number the eigenvalues $\left\{\nu_{k}\right\}$ of the operator $L$ in nonincreasing order, taking into account multiplicity. Then the orthonormal (in the sense of $\mathfrak{Y}$ ) family of corresponding eigenfunctions $\left\{\psi_{k}\right\}$ of operator $L$ forms the basis in $\mathfrak{X}$. 
For $\lambda \in \mathbb{R}_{+}$construct the operator $A=\lambda \mathbb{I}+L$. By construction operator $A \in \mathcal{L}(\mathfrak{X} ; \mathfrak{Y})$, and its spectrum $\sigma(A)=\left\{\lambda+\nu_{k}\right\}$. Define the operator $B$ by formula $B=\alpha \mathbb{I}$, where $\alpha \in \mathbb{R}$, and $C=\mathbb{I}$. Thus, we reduced problem $(3)-(5)$ to equation (10). Construct the relative spectrum of the operator $B$

$$
\sigma^{A}(B)=\left\{\mu_{k}=\frac{\alpha_{j}}{\lambda+\nu_{k}}: k \in \mathbb{N} \backslash\left\{l: \lambda+\nu_{k}=0\right\}\right\} .
$$

Lemma 4. [6] The operator $B$ is $(A, 0)$-bounded if one of the following conditions holds:

(i) $\operatorname{ker} A=\{0\}, \lambda \neq \nu_{k}, \forall k \in \mathbb{N}$;

(ii) $\operatorname{ker} A \neq\{0\}, \alpha_{j} \neq 0$ for any $j$.

Further, consider the initial-final problem for mathematical model (3) - (5). Represent the $A$-spectrum of operator $B$ in the form

$$
\sigma^{A}(B)=\sigma_{i n}^{A}(B) \cup \sigma_{\text {fin }}^{A}(B), \sigma_{\text {in }}^{A}(B) \cap \sigma_{\text {fin }}^{A}(B)=\emptyset .
$$

Then the initial-final condition (13) takes the form

$$
\sum_{\mu_{k} \in \sigma_{\text {in }}^{A}(B)}\left\langle\left(x(0)-x_{0}\right), \psi_{k}\right\rangle \psi_{k}=0, \sum_{\mu_{k} \in \sigma_{\text {fin }}^{A}(B)}\left\langle\left(x(\tau)-x_{\tau}\right), \psi_{k}\right\rangle \psi_{k}=0 .
$$

Introduce the control space

$$
H^{1}(\mathfrak{U})=\left\{u=\left(u_{1}, u_{2}, \ldots u_{j}, \ldots\right): \dot{u}_{j} \in L_{2}\left(0, \tau ;\left(0, l_{j}\right)\right)\right\},
$$

and choose a closed convex subset $\mathfrak{U}_{a d}$ in it. Construct the operators

$$
\left\langle N_{q} u^{(q)}, u^{(q)}\right\rangle=\sum_{E_{j} \in \mathfrak{E}} d_{j} \int_{0}^{l_{j}} \varkappa_{j q}\left(u_{j}^{(q)}\right)^{2} d s,
$$

where $\varkappa_{j q}$ are positive numbers.

Theorem 11. [8] For any $y \in H^{1}(\mathfrak{Y})$ and $\lambda \in \mathbb{R}_{+}, \alpha_{j} \in \mathbb{R}$, satisfying condition (i) or (ii) of Lemma 4 , for arbitrary $x_{0}, x_{\tau} \in \mathfrak{X}$ there exists a unique solution $(\hat{x}, \hat{u}) \in H^{1}(\mathfrak{X}) \times \mathfrak{U}_{a d}$ of optimal control problem (14) for mathematical model (3) - (5) with initial-final conditions (26), that minimizes functional (20).

Optimal control in the Boussinesq-Löve model. Consider the optimal control problem for mathematical model (1), (6) with the initial Showalter-Sidorov condition (12). Reduce the problem to equation (10) with $n=2$. To do this, put

$$
\mathfrak{X}=\left\{x \in H^{l+2}(\Omega): x(s)=0, s \in \partial \Omega\right\}, \quad \mathfrak{Y}=H^{l}(\Omega), \quad \mathfrak{U}=H^{l}(\Omega), \quad l \in\{0\} \cup \mathbb{N} .
$$

The operators $A, B \in \mathcal{L}(\mathfrak{X} ; \mathfrak{Y})$ are given by formulas $A=\lambda \mathbb{I}-\Delta, B=\beta\left(\Delta-\lambda^{\prime}\right), C=\mathbb{I}$. Then the $A$-resolvent of operator $B$ takes the form

$$
\left(\mu^{2} A-B\right)^{-1}=\sum_{k=1}^{\infty} \frac{\left\langle\cdot, \varphi_{k}\right\rangle \varphi_{k}}{\left(\lambda-\lambda_{k}\right) \mu^{2}+\beta\left(\lambda^{\prime}-\lambda_{k}\right)},
$$


where $\langle\cdot, \cdot\rangle$ is an inner product in $L_{2}(\Omega), \lambda_{k}, \varphi_{k}$ are the same as in Barenblatt-ZheltovKochina model. Then for the $A$-spectrum of operator $B$ we get

$$
\sigma^{A}(B)=\left\{\mu_{k}^{2}=-\frac{\beta\left(\lambda^{\prime}-\lambda_{k}\right)}{\lambda-\lambda_{k}}: \lambda \neq \lambda_{k}\right\}
$$

Lemma 5. [10] The operator $B$ is $(A, 0)$-bounded.

Construct the projector:

$$
P=\left\{\begin{array}{l}
\mathbb{I}, \text { if } \lambda \neq \lambda_{k} \\
\mathbb{I}-\sum_{\lambda_{k}=\lambda}\left\langle\cdot, \varphi_{k}\right\rangle \varphi_{k}, \text { if } \lambda_{k}=\lambda
\end{array}\right.
$$

The Showalter-Sidorov condition (12) is given by

$$
\sum_{\lambda_{k} \neq \lambda}<\varphi_{k}, x(s, 0)-x_{0}(s)>\varphi_{k}=0, \sum_{\lambda_{k} \neq \lambda}<\varphi_{k}, x_{t}(s, 0)-x_{1}(s)>\varphi_{k}=0 .
$$

Let us proceed to the optimal control problem. Introduce the control space

$$
H^{2}(\mathfrak{U})=\left\{u \in L_{2}(0, \tau ; \mathfrak{U}): \ddot{u} \in L_{2}(0, \tau ; \mathfrak{U})\right\} .
$$

In the space $H^{2}(\mathfrak{U})$ single out a closed convex subset $\mathfrak{U}_{a d}$, which will be the set of admissible controls.

Theorem 12. [10] For any $\alpha, \beta \in \mathbb{R} \backslash\{0\}, \lambda \in \mathbb{R}$, and $\tau \in \mathbb{R}_{+}, x_{m} \in \mathfrak{X}, m=0,1$, there exists a unique solution $(\hat{x}, \hat{u}) \in H^{2}(\mathfrak{X}) \times \mathfrak{U}_{a d}$ to the optimal control problem (14) for the Boussinesq-Löve model (1), (6) with the Showalter-Sidorov condition (29), that minimizes functional (20).

\section{Relatively $p$-Sectorial Operators}

In this section, definitions and results of the theory of relatively sectorial operators are given $[3$, Ch. 3]. Let $\mathfrak{X}, \mathfrak{Y}, \mathfrak{U}$ be Banach spaces, operators $A \in \mathcal{L}(\mathfrak{X} ; \mathfrak{Y}), B \in \mathcal{C} l(\mathfrak{X} ; \mathfrak{Y})$, $C \in \mathcal{L}(\mathfrak{U} ; \mathfrak{Y})$, functions $y:(0, \tau) \subset \mathbb{R}_{+} \rightarrow \mathfrak{Y}(\tau<\infty)$ to be determined later. Let further $\mu_{k} \in \rho^{A}(B), k=0,1, \ldots, p$. Operator-functions

$$
R_{(\mu, p)}^{A}=\prod_{k=0}^{p} R_{\mu_{k}}^{A}(B), \quad L_{(\mu, p)}^{A}(B)=\prod_{k=0}^{p} L_{\mu_{k}}^{A}(B)
$$

are called, respectively, a right and a left $(A, p)$-resolvents of operator $B$.

Definition 8. [3, Ch. 3] The operator $B$ is called p-sectorial with respect to the operator $A$ with the number $p \in\{0\} \cup \mathbb{N}$ (or simply $(A, p)$-sectorial for some $p \in\{0\} \cup \mathbb{N}$ ) if

(i) there are constants $a \in \mathbb{R}$ and $\Theta \in\left(\frac{\pi}{2}, \pi\right)$ such that

$$
S_{a, \Theta}^{A}(B)=\{\mu \in \mathbb{C}:|\arg (\mu-a)|<\Theta, \mu \neq a\} \subset \rho^{A}(B),
$$


(ii) there is a constant $K \in \mathbb{R}_{+}$such that

$$
\max \left\{\left\|R_{(\mu, p)}^{L}(B)\right\|_{\mathcal{L}(\mathfrak{X})},\left\|L_{(\mu, p)}^{A}(B)\right\|_{\mathcal{L}(\mathfrak{Y})}\right\} \leq \frac{K}{\prod_{q=0}^{p}\left|\mu_{q}-a\right|},
$$

for any $\mu_{q} \in S_{a, \Theta}^{A}(B), q=0,1, \ldots, p$.

Let the operator $B$ be $(A, p)$-sectorial for some $p \in\{0\} \cup \mathbb{N}$, then there are degenerate analytic semigroups of operators (see [3, Ch. 3])

$$
X^{t}=\frac{1}{2 \pi i} \int_{\Gamma} R_{\mu}^{A}(B) e^{\mu t} d \mu \quad \text { and } \quad Y^{t}=\frac{1}{2 \pi i} \int_{\Gamma} L_{\mu}^{A}(B) e^{\mu t} d \mu
$$

where $t \in \mathbb{R}_{+}$, and the contour $\Gamma \subset S_{a, \Theta}^{A}(B)$ is such that $|\arg \mu| \rightarrow \Theta$ for $\mu \rightarrow \infty, \mu \in \Gamma$, $\Theta \in\left(\frac{\pi}{2}, \pi\right)$. Denote by $X^{\bullet}=\left\{X^{t}: t \in \mathbb{R}_{+}\right\}$. Put $\mathfrak{X}^{0}=\operatorname{ker} X^{\bullet}, \mathfrak{X}^{1}=\operatorname{im} X^{\bullet}, \mathfrak{Y}^{0}=\operatorname{ker} Y^{\bullet}$, $\mathfrak{Y}^{1}=\operatorname{im} Y^{\bullet}$ and denote by $A_{k}$ the restriction of operator $A$ to $\mathfrak{X}^{k}$, and by $B_{k}$ the restriction of operator $B$ to $\mathfrak{X}^{k} \cap \operatorname{dom} B, k=0,1$.

There are two approaches to splitting of spaces $\mathfrak{X}$ and $\mathfrak{Y}$. The first approach is outlined in [3], where a stronger condition on operator $B$ (strong $(A, p)$-sectoriality of operator $B)$ is set. We follow the equivalent approach firstly proposed by G.A. Sviridyuk. Further, we need two conditions:

$$
\begin{gathered}
\mathfrak{X}^{0} \oplus \mathfrak{X}^{1}=\mathfrak{X} \text { and } \mathfrak{Y}^{0} \oplus \mathfrak{Y}^{1}=\mathfrak{Y}, \\
\text { there exists an operator } A_{1}^{-1} \in \mathcal{L}\left(\mathfrak{Y}^{1} ; \mathfrak{X}^{1}\right) .
\end{gathered}
$$

The equivalence of these approaches was shown in [33]. Note that condition $(A 1)$ occurs when the operator $B$ is strongly $(A, p)$-sectorial on the right (left). Condition $(A 2)$ is met either when the operator $B$ is strongly $(A, p)$-sectorial, or when it is $(A, p)$-sectorial, $(A 1)$ holds and $\mathfrak{Y}^{1}=\operatorname{im} A_{1}$. If conditions $(A 1),(A 2)$ are satisfied and operator $B$ is $(A, p)$ sectorial, then there are projectors $P=s$ - $\lim _{t \rightarrow 0+} X^{t}, Q=s$ - $\lim _{t \rightarrow 0+} Y^{t}$, operators $H=B_{0}^{-1} A_{0} \in$ $\mathcal{L}\left(\mathfrak{X}^{0}\right)$ and $S=A_{1}^{-1} B_{1} \in \mathcal{C l}\left(\mathfrak{X}^{1}\right)$. Moreover, the operator $H$ is nilpotent of degree $p$, and the operator $S$ is sectorial.

Further we consider the case $n=1$ and equation (10) in the form

$$
A \dot{x}=B x+y
$$

Proceed to the study of solvability of the Cauchy problem (11) for equation (30). Similarly to Section 1 for the solvability of the Cauchy problem, it is necessary to construct a condition connecting the initial value and $y$ in the right-hand side of (30). Introduce the set

$$
\mathcal{M}_{y}=\left\{x \in \mathfrak{X}:(\mathbb{I}-P) x+\sum_{q=0}^{p} H^{q} B_{0}^{-1}(\mathbb{I}-Q) y^{(q)}(0)=0\right\} .
$$

The solvability of the Cauchy problem (11), (30) was studied in [3,13].

Theorem 13. [3,13] Let the operator $B$ be $(A, p)$-sectorial for some $p \in\{0\} \cup \mathbb{N}$, conditions (A1), (A2) be fulfilled and $y^{0}=(\mathbb{I}-Q) y \in C^{p}\left([0, \tau] ; \mathfrak{Y}^{0}\right) \cap C^{p+1}\left((0, \tau) ; \mathfrak{Y}^{0}\right), y^{1}=Q y \in$ 
$C\left([0, \tau] ; \mathfrak{Y}^{1}\right)$. Then for any $x_{0} \in \mathcal{M}_{y}$ there exists a unique classical solution to (11), (30) given by

$$
x(t)=X^{t} x_{0}-\sum_{q=0}^{p} H^{q} B_{0}^{-1}(\mathbb{I}-Q) y^{(q)}(t)+\int_{0}^{t} X^{t-s} y(s) d s .
$$

Proceed to more general initial-final condition (13) and the Showalter-Sidorov condition (12). If we use these conditions, we can omit conditions on the initial value $x_{0}$, and take it from the whole space $\mathfrak{X}$. Suppose that conditions $(A 1),(B)$ are satisfied. Construct a relatively spectral projectors [4]

$$
P_{\text {fin }}=\frac{1}{2 \pi i} \int_{\gamma_{0}} R_{\mu}^{A}(B) d \mu, \quad Q_{\text {fin }}=\frac{1}{2 \pi i} \int_{\gamma_{0}} L_{\mu}^{A}(B) d \mu .
$$

It turns out that under $(A, p)$-sectorial for some $p \in\{0\} \cup \mathbb{N}$ and conditions $(A 1),(B)$ being fulfilled $P_{f i n} P=P P_{f i n}=P_{f i n}, Q_{f i n} Q=Q Q_{f i n}=Q_{f i n}$. So there exist projectors

$$
P_{i n}=P-P_{f i n}, \quad Q_{i n}=Q-Q_{f i n} .
$$

Then $\mathfrak{Y}^{\text {in }}=\mathrm{im} \mathrm{Q}_{\text {in }}, \mathfrak{Y}^{\text {fin }}=\mathrm{im} \mathrm{Q}_{\text {fin }}$.

Theorem 14. [14] Let the operator $B$ be $(A, p)$-sectorial for some $p \in\{0\} \cup \mathbb{N}$ and conditions $(A 1),(A 2),(B)$ be satisfied. Then for any $x_{0}, x_{\tau} \in \mathfrak{X}$ and vector function $y$ such that $y^{0}=(\mathbb{I}-Q) y \in C^{p}\left([0, \tau] ; \mathfrak{Y}^{0}\right) \cap C^{p+1}\left((0, \tau) ; \mathfrak{Y}^{0}\right), y^{\text {in }}=Q_{\text {in }} y \in C\left([0, \tau] ; \mathfrak{Y}^{\text {in }}\right)$, $y^{\text {fin }}=Q_{\text {fin }} y \in C\left([0, \tau] ; \mathfrak{Y}^{\text {fin }}\right)$ there exists a unique classical solution to (13), (30), given by

$$
\begin{gathered}
x(t)=-\sum_{q=0}^{p} H^{q} B_{0}^{-1} \frac{d^{q}}{d t^{q}} y^{0}(t)+X_{\text {in }}^{t} x_{0}+\int_{0}^{t} Z_{\text {in }}^{t-s} y^{i n}(s) d s+ \\
+X_{\text {fin }}^{t-\tau} x_{\tau}-\int_{t}^{\tau} Z_{\text {fin }}^{t-s} y^{f i n}(s) d s
\end{gathered}
$$

where

$$
\begin{gathered}
X_{i n}^{t}=\frac{1}{2 \pi i}\left(\int_{\Gamma} R_{\mu}^{A}(B) e^{\mu t} d \mu-\int_{\gamma_{0}} R_{\mu}^{A}(B) e^{\mu t} d \mu\right), \\
Z_{\text {in }}^{t}=\frac{1}{2 \pi i}\left(\int_{\Gamma}(\mu A-B)^{-1} e^{\mu t} d \mu-\int_{\gamma_{0}}(\mu A-B)^{-1} e^{\mu t} d \mu\right), \\
X_{\text {fin }}^{t}=\frac{1}{2 \pi i} \int_{\gamma} R_{\mu}^{A}(B) e^{\mu t} d \mu, \quad Z_{\text {fin }}^{t}=\frac{1}{2 \pi i} \int_{\gamma_{0}}(\mu A-B)^{-1} e^{\mu t} d \mu .
\end{gathered}
$$

Corollary 3. Let the operator $B$ be $(A, p)$-sectorial for some $p \in\{0\} \cup \mathbb{N}$, conditions $(A 1)$, $(A 2)$ be fulfilled and $y \in C^{p}((0, \tau) ; \mathfrak{X}) \cap C^{p+1}([0, \tau] ; \mathfrak{X})$. Then for any $x_{0} \in \mathfrak{X}$ there exists a unique classical solution to the Showalter-Sidorov problem (12), (30) given by (32).

Next, we consider the optimal control problem. Let now $\mathfrak{X}, \mathfrak{Y}$ and $\mathfrak{U}$ be Hilbert spaces. Similarly to Section 1, consider strong solutions to

$$
A \dot{x}=B x+y+C u .
$$


Definition 9. The vector-function $x \in H^{1}(\mathfrak{X})=\left\{x \in L_{2}(0, \tau ; \mathfrak{X}): \dot{x} \in L_{2}(0, \tau ; \mathfrak{X})\right\}$ is called a strong solution to (33), if it turns the equation to an identity almost everywhere on interval $(0, \tau)$. A strong solution $x=x(t)$ of (33) is called

- a strong solution of the Cauchy problem if $\lim _{t \rightarrow 0+}\left(x(t)-x_{0}\right)=0$;

- a strong solution of the Showalter-Sidorov problem if $\lim _{t \rightarrow 0+} P\left(x(t)-x_{0}\right)=0$;

- a strong solution of the initial-final problem if $\lim _{t \rightarrow 0+} P_{i n}\left(x(t)-x_{0}\right)=0$ and $P_{f i n}(x(\tau)-$ $\left.x_{\tau}\right)=0$.

Similarly to Section 1, there exists a unique strong solution to the Cauchy problem (11), (30) [13]. This implies that the set of admissible pairs $(x, u)$ is non-empty. When using the Cauchy condition, we pass to the consideration of the subspace of controls

$$
\stackrel{\circ}{H}^{p+1}(\mathfrak{U})=\left\{u \in L_{2}(0, \tau ; \mathfrak{U}): u^{(q)} \in L_{2}(0, \tau ; \mathfrak{U}), u^{(q)}(0)=0, q=\overline{0, p+1}\right\}, p \in\{0\} \cup \mathbb{N} .
$$

In the space $\stackrel{\circ}{H}^{p+1}(\mathfrak{U})$ we single out a closed convex subset $\mathfrak{U}_{a d} \subset \stackrel{\circ}{H}^{p+1}(\mathfrak{U})$.

Theorem 15. [13] Let the operator $B$ be $(A, p)$-sectorial for some $p \in\{0\} \cup \mathbb{N}$ and conditions (A1), (A2) be fulfilled. Then for any $y \in H^{p+1}(\mathfrak{Y}), x_{0} \in \mathcal{M}_{y}$ there exists a unique solution to the optimal control problem (11), (14), (33) with functional (20).

Proceed to consideration of more general initial-final condition (13) and the ShowalterSidorov condition (12). Introduce the control space

$$
H^{p+1}(\mathfrak{U})=\left\{u \in L_{2}(0, \tau ; \mathfrak{U}): u^{(q)} \in L_{2}(0, \tau ; \mathfrak{U}), q=\overline{0, p+1}\right\}, p \in\{0\} \cup \mathbb{N}
$$

and select a closed convex subset $\mathfrak{U}_{a d} \subset H^{p+1}(\mathfrak{U})$.

Theorem 16. [8] Let the operator $B$ be $(A, p)$-sectorial for some $p \in\{0\} \cup \mathbb{N}$ and conditions $(A 1),(A 2),(B)$ be fulfilled. Then for any $y \in H^{p+1}(\mathfrak{Y}), x_{0}, x_{\tau} \in \mathfrak{X}$ there exists a unique solution to the optimal control problem (13), (14), (33) with functional (20).

Corollary 4. Let the operator $B$ be $(A, p)$-sectorial for some $p \in\{0\} \cup \mathbb{N}$ and conditions (A1), (A2) be fulfilled. Then for any $y \in H^{p+1}(\mathfrak{Y}), x_{0} \in \mathfrak{X}$ there exists a unique solution to the optimal control problem (12), (14), (33) with functional (20).

\section{Optimal Control in Sobolev-Type Models with Relatively $p$-Sectorial Operators}

Optimal control in the Dzektzer model. Consider the Dzektser model (7), (8). For reducing problem (7), (8) to equation (33), put

$$
\mathfrak{X}=\left\{x \in H^{l+2}(\Omega): x(s)=0, s \in \partial \Omega\right\}, \quad \mathfrak{Y}=\mathfrak{U}=H^{l}(\Omega), \quad l=0,1, \ldots,
$$

and define the operators $A, B$ by the formulas

$$
A=\lambda \mathbb{I}-\Delta, \quad B=\alpha \Delta-\beta \Delta^{2}+\gamma \mathbb{I}, \quad C=\mathbb{I} .
$$

Construct the set

$$
\operatorname{dom} B=\left\{x \in H^{l+4}(\Omega): \Delta x(s)=0, s \in \partial \Omega\right\} \cap \mathfrak{X} .
$$


By definition, the operator $A \in \mathcal{L}(\mathfrak{X} ; \mathfrak{Y})$, and the operator $B \in \mathcal{C l}(\mathfrak{X}, \mathfrak{Y})$. The relative spectrum of the operator $B$ is given by

$$
\sigma^{A}(B)=\left\{\mu_{k}=\frac{\alpha \lambda_{k}-\beta \lambda_{k}^{2}+\gamma}{\lambda-\lambda_{k}}: \lambda \neq \lambda_{k}\right\}
$$

where $\lambda_{k}, \varphi_{k}$ are the same as in the Barenblatt-Zheltov-Kochina model.

Lemma 6. [13] For any $\alpha \in \mathbb{R}_{+}$and $\beta, \gamma, \lambda \in \mathbb{R}$ such that either $-\lambda \notin \sigma(A)$, or $-\lambda \in \sigma(A)$ and $-\lambda$ is not the root of the equation $\alpha a-\beta a^{2}+\gamma=0$, the operator $M$ is strongly $(L, 0)$-sectorial.

Consider the set

$$
\mathcal{M}_{y}=\left\{x \in \mathfrak{X}:\left(-\alpha \lambda+\beta \lambda^{2}-\gamma\right)\left\langle x, \varphi_{k}\right\rangle=\left\langle y(0), \varphi_{k}\right\rangle, \lambda_{k}=\lambda\right\},
$$

where $\langle\cdot, \cdot\rangle$ is an inner product in $L_{2}(\Omega)$. Introduce the control space

$$
\stackrel{\circ}{H}^{1}(\mathfrak{U})=\left\{u \in L_{2}(0, \tau ; \mathfrak{U}): \dot{u} \in L_{2}(0, \tau ; \mathfrak{U}), u(0)=0\right\}
$$

and construct the penalty functional (20). Choose a closed and convex subset $\mathfrak{U}_{a d} \subset \stackrel{\circ}{H}^{1}(\mathfrak{U})$.

Theorem 17. [13] Let the conditions of Lemma 6 be satisfied. Then for any $y \in H^{1}(\mathfrak{Y})$ and $x \in \mathcal{M}_{y}$ there exists a unique optimal control $u \in \mathfrak{U}_{a d}$ of solutions to the Cauchy problem (11) for the model (7), (8), minimizing functional (20).

Optimal control in the Dzektser model on the graph. Let $\mathbf{G}=\mathbf{G}(\mathfrak{V} ; \mathfrak{E})$ be a finite connected oriented graph, where $\mathfrak{V}=\left\{V_{i}\right\}$ is a set of vertices, and $\mathfrak{E}=\left\{E_{i}\right\}$ is a set of edge, and each edges $E_{i}$ has the length $l_{i} \in \mathbb{R}_{+}$and the cross sectional area $d_{i} \in \mathbb{R}_{+}$. On the graph $\mathbf{G}$ consider linear partial differential equations

$$
\lambda x_{j t}-x_{j t s s}=\beta x_{j s s}-\alpha x_{j s s s}+\gamma x_{j}+u_{j} .
$$

We look for a solution to equation (34) on the graph $\mathbf{G}$, satisfying the continuity conditions (3) and flow balance condition (4).

Reduce the mathematical model (3), (4), (34) to equation (33). Let $\lambda \in \mathbb{R}$ and define the operator $A=\lambda \mathbb{I}-L$, where $L$ is defined by $(25)$ and the space $\mathfrak{X}, \mathfrak{Y}, \mathfrak{U}$ are the same as in the Hoff model on a graph. By construction the operator $A \in \mathcal{L}(\mathfrak{X} ; \mathfrak{Y})$, and its spectrum $\sigma(A)=\left\{\lambda-\nu_{k}\right\}$. Consider the set

$$
\begin{gathered}
\operatorname{dom} B=\left\{x \in \mathfrak{X}: x_{j} \in H^{4}\left(0, l_{j}\right)\right. \text { and } \\
x_{j s s}(0, t)=x_{k s s}(0, t)=x_{m s s}\left(l_{m}, t\right)=x_{n s s}\left(l_{n}, t\right), \quad E_{j}, E_{k} \in E^{\alpha}\left(V_{i}\right), E_{m}, E_{n} \in E^{\omega}\left(V_{i}\right) ; \\
\left.\sum_{E_{j} \in E^{\alpha}\left(V_{i}\right)} d_{j} x_{j s s s}(0, t)-\sum_{E_{k} \in E^{\omega}\left(V_{i}\right)} d_{k} x_{k s s}\left(l_{k}, t\right)=0\right\} .
\end{gathered}
$$

Further, the formula $W: v \rightarrow\left(v_{1 s s s s}, v_{2 s s s}, \ldots, v_{j s s s s}, \ldots\right)$ defines the operator $W \in \mathcal{L}(\operatorname{dom} B ; \mathfrak{Y})$ and $\sigma(W)=\left\{\nu_{k}^{2}\right\}$. Take $\beta, \gamma, \lambda \in \mathbb{R}$ and define an operator $B=-\beta W+\alpha L+\gamma \mathbb{I}$. By construction $B \in \mathcal{C l}(\mathfrak{X} ; \mathfrak{Y})$. Let $\sigma^{A}(B)=\sigma_{\text {in }}^{A}(B) \cup \sigma_{\text {fin }}^{A}(B)$, $\sigma_{i n}^{A}(B) \cap \sigma_{\text {fin }}^{A}(B)=\emptyset$. Then condition (13) takes the form

$$
\lim _{t \rightarrow 0+} \sum_{\mu_{k} \in \sigma_{1}^{A}(B)}\left\langle\left(x(t)-x_{0}\right), \psi_{k}\right\rangle \psi_{k}=0, \sum_{\mu_{k} \in \sigma_{0}^{A}(B)}\left\langle\left(x(\tau)-x_{\tau}\right), \psi_{k}\right\rangle \psi_{k}=0,
$$


where $\nu_{k}, \psi_{k}$ are the same as in the Hoff model on a graph. Thus, we reduced problem (3), (4), (34), (35) to problem (13), (33).

Introduce the space

$$
H^{1}(\mathfrak{U})=\left\{u=\left(u_{1}, u_{2}, \ldots u_{j}, \ldots\right): \dot{u}_{j} \in L_{2}\left(0, \tau ;\left(0, l_{j}\right)\right)\right\} .
$$

Single out a closed convex subset $\mathfrak{U}_{a d} \subset H^{1}(\mathfrak{U})$. Construct the operators

$$
\left\langle N_{q} u^{(q)}, u^{(q)}\right\rangle=\sum_{E_{j} \in \mathfrak{E}} d_{j} \int_{0}^{l_{j}} \varkappa_{j q}\left(u_{j}^{(q)}\right)^{2} d s,
$$

where $\varkappa_{j q}$ are positive numbers.

Theorem 18. Let the conditions of Lemma 6 be satisfied. Then for any $\tau \in \mathbb{R}_{+}, x_{0}, x_{\tau} \in \mathfrak{X}$ there exists a unique optimal control $u \in \mathfrak{U}_{a d}$ of solutions to the initial-final problem (35) for the model (3), (4), (34), minimizing functional (20).

\section{Relatively $p$-Radial Operators}

In this section, definitions and results of the theory of relatively radial operators are given [3, Ch. 2]. Let $\mathfrak{X}, \mathfrak{Y}, \mathfrak{U}$ be Banach spaces, and the operators $A \in \mathcal{L}(\mathfrak{X} ; \mathfrak{Y})$, $B \in \mathcal{C l}(\mathfrak{X} ; \mathfrak{Y}), C \in \mathcal{L}(\mathfrak{Y}) ; \mathfrak{U})$.

Definition 10. The operator $B$ is called $p$-radial for some $p \in\{0\} \cup \mathbb{N}$, with respect to operator $A((A, p)$-radial $)$, if

(i) $\exists a \in \mathbb{R} \forall \mu>a \mu \in \rho^{A}(B)$;

(ii) $\exists K>0 \forall \mu=\left(\mu_{0}, \mu_{1}, \ldots, \mu_{p}\right) \in(a,+\infty)^{p+1} \forall n \in \mathbb{N}$

$$
\max \left\{\left\|\left(R_{(\mu, p)}^{A}(B)\right)^{n}\right\|_{\mathcal{L}(\mathfrak{X})},\left\|\left(L_{(\mu, p)}^{A}(B)\right)^{n}\right\|_{\mathcal{L}(\mathfrak{Y})}\right\} \leq \frac{K}{\prod_{k=0}^{p}\left(\mu_{k}-a\right)^{n}} .
$$

Whereof, we assume that the operator $B$ is $(A, p)$-radial for some $p \in\{0\} \cup \mathbb{N}$. Put $\mathfrak{X}^{0}=\operatorname{ker} R_{(\mu, p)}^{A}(B), \mathfrak{Y}^{0}=\operatorname{ker} L_{(\mu, p)}^{A}(B)$. By $A_{0}$ denote the restriction of the operator $A$ on $\mathfrak{X}^{0}$, and by $B_{0}$ denote the restriction of the operator $B$ on $\mathfrak{X}^{0} \cap \operatorname{dom} B$.

Theorem 19. [3, Ch. 2] Let the operator $B$ be $(A, p)$-radial for some $p \in\{0\} \cup \mathbb{N}$. Then

(i) the operator $A_{0} \in \mathcal{L}\left(\mathfrak{X}^{0} ; \mathfrak{Y}^{0}\right)$ and the operator $B_{0} \in \mathcal{C l}\left(\mathfrak{X}^{0} ; \mathfrak{Y}^{0}\right)$;

(ii) there exists the operator $B_{0}^{-1} \in \mathcal{L}\left(\mathfrak{Y}^{0} ; \mathfrak{X}^{0}\right)$;

(iii) the operator $H=B_{0}^{-1} A_{0} \in \mathcal{L}\left(\mathfrak{X}^{0}\right)$ is nilpotent with degree less than or equal to $p$.

Let $\mathfrak{X}^{1}$ be the closure of the lineal im $R_{(\mu, p)}^{A}(B)$, and $\mathfrak{Y}^{1}$ be the closure of the lineal $\operatorname{im} L_{(\mu, p)}^{A}(B)$. Let $\tilde{\mathfrak{X}}$ be the closure of the lineal $\mathfrak{X}^{0} \dot{+} \operatorname{im} R_{(\mu, p)}^{A}(B)$ in the norm of the space $\mathfrak{X}$, and $\tilde{\mathfrak{Y}}$ be the closure of the lineal $\mathfrak{Y}^{0} \dot{+} \operatorname{im} L_{(\mu, p)}^{A}(B)$ in the norm of the space $\mathfrak{Y}$. By $A_{1}$ denote the restriction of the operator $A$ on $\mathfrak{X}^{1}$, and by $B_{1}$ denote the restriction of the operator $B$ on $\mathfrak{X}^{1} \cap \operatorname{dom} B$.

Theorem 20. [3, Ch. 2] Let the operator $B$ be $(A, p)$-radial for some $p \in\{0\} \cup \mathbb{N}$. Then there exists a uniformly bounded and strongly continuous resolving semigroup of equation 
(30) on the subspace $\tilde{\mathfrak{X}}(\tilde{\mathfrak{Y}})$ given by

$$
\begin{gathered}
X^{t}=s-\lim _{k \rightarrow \infty}\left(\left(A-\frac{t}{k} B\right)^{-1} L\right)^{k}=s-\lim _{k \rightarrow \infty}\left(\frac{k}{t} R_{\frac{k}{t}}^{A}(B)\right)^{k} \\
\left(Y^{t}=s-\lim _{k \rightarrow \infty}\left(A\left(A-\frac{t}{k} B\right)^{-1}\right)^{k}=s-\lim _{k \rightarrow \infty}\left(\frac{k}{t} L_{\frac{k}{t}}^{A}(B)\right)^{k}\right) .
\end{gathered}
$$

Similarly to Section 3 , we introduce the conditions $(A 1),(A 2),(B)$. Whereof, we assume that conditions $(A 1),(B)$ are fulfilled, then construct projectors [4]

$$
P_{\text {fin }}=\frac{1}{2 \pi i} \int_{\gamma} R_{\mu}^{A}(B) d \mu, \quad Q_{\text {fin }}=\frac{1}{2 \pi i} \int_{\gamma} L_{\mu}^{A}(B) d \mu .
$$

There exist projectors

$$
P_{i n}=P-P_{f i n}, \quad Q_{i n}=Q-Q_{f i n} .
$$

Let $\mathfrak{X}_{i n(f i n)}^{1}=\operatorname{im} P_{i n(f i n)}, \mathfrak{Y}_{i n(f i n)}^{1}=\operatorname{im} Q_{i n(f i n)}$. By $A_{i n(f i n)}$ denote the restriction of the operator $A$ on subspace $\mathfrak{X}_{i n(f i n)}^{1}$, by $B_{\text {in (fin) }}$ denote the restriction of the operator $B$ on subspace $\mathfrak{X}_{i n(f i n)}^{1} \cap \operatorname{dom} B$. Similarly to Section 3 actions of the operators split, i.e. the operator $A_{\text {in }(\text { fin })} \in \mathcal{L}\left(\mathfrak{X}_{\text {in(fin })}^{1} ; \mathfrak{Y}_{\text {in(fin })}^{1}\right)$; the operators $A_{\text {in (fin })}^{-1} \in \mathcal{L}\left(\mathfrak{Y}_{\text {in (fin })}^{1}, B_{\text {in }(\text { fin })} \in\right.$ $\left.\mathcal{C l}\left(\mathfrak{X}_{i n(f i n)}^{1} ; \mathfrak{Y}_{i n(f i n)}^{1}\right) ; \mathfrak{X}_{i n(f i n)}^{1}\right)$ exist.

Theorem 21. [16] Let the operator $B$ be $(A, p)$-radial for some $p \in\{0\} \cup \mathbb{N}$ and conditions $(A 1),(A 2),(B)$ be fulfilled. Then for all $x_{0}, x_{\tau} \in \mathfrak{X}$ and the vector function $y:[0, \tau] \rightarrow \mathfrak{Y}$, such that $y^{0}=(\mathbb{I}-Q) y \in C^{p+1}\left([0, \tau] ; \mathfrak{Y}^{0}\right), y_{\text {in }}^{1}=Q_{\text {in }} y \in C\left([0, \tau] ; \mathfrak{Y}_{\text {in }}^{1}\right), y_{\text {fin }}^{1}=Q_{\text {fin }} y \in$ $C\left([0, \tau] ; \mathfrak{Y}_{\text {fin }}^{1}\right)$ there exists a unique classical solution $x \in C([0, \tau] ; \mathfrak{X}) \cap C^{1}((0, \tau) ; \mathfrak{X})$ of (13), (30) given by

$$
\begin{gathered}
x(t)=-\sum_{q=0}^{p}\left(B_{0}^{-1} A_{0}\right)^{q} B_{0}^{-1} \frac{d^{q}}{d t^{q}} y^{0}(t)+X_{\text {in }}^{t} x_{0}+\int_{0}^{t} R_{i n}^{t-s} y_{\text {in }}^{1}(s) d s+X_{f i n}^{t-\tau} x_{\tau}- \\
-\int_{t}^{\tau} R_{\text {fin }}^{t-s} y_{\text {fin }}^{1}(s) d s,
\end{gathered}
$$

where

$R_{f i n}^{t}=\frac{1}{2 \pi i} \int_{\gamma}\left(\mu A_{f i n}-B_{f i n}\right)^{-1} e^{\mu t} d \mu, R_{i n}^{t}=s-\lim _{k \rightarrow \infty}\left(\left(A_{i n}-\frac{t}{k} B_{i n}\right)^{-1} A_{i n}\right)^{k}\left(A_{i n}-\frac{t}{k} B_{i n}\right)^{-1}$.

Let $\mathfrak{X}, \mathfrak{Y}, \mathfrak{U}$ be Hilbert spaces. Consider the control space $H^{p+1}(\mathfrak{U})=\left\{u \in L_{2}(0, \tau ; \mathfrak{U})\right.$ : $\left.u^{(p+1)} \in L_{2}(0, \tau ; \mathfrak{U})\right\}$. Choose the closed and convex subset $\mathfrak{U}_{a d}$ in the space $H^{p+1}(\mathfrak{U})$, which is called the set of admissible controls.

Theorem 22. [16] Let the operator $B$ be $(A, p)$-radial for some $p \in\{0\} \cup \mathbb{N}$ and conditions $(A 1),(A 2),(B)$ be fulfilled. For all $y \in H^{p+1}(\mathfrak{Y}), x_{0}, x_{\tau} \in \mathfrak{X}$ there exists a unique optimal control of solutions of problem (13), (33). 


\section{Optimal Control in Sobolev-type Models with a Relatively $p$-Radial Operator}

Optimal control in the Chen-Gurtin model. Define the spaces $\mathfrak{X}=H^{2}(\Omega) \cap \stackrel{\circ}{H^{1}}(\Omega)$, $\mathfrak{Y}=L_{2}(\Omega)$ and $\mathfrak{U}=L_{2}(\Omega)$. Reduce (7), (9) to equation (33). Fix $\nu, d, \lambda \in \mathbb{R}$, define the operators $A=\lambda \mathbb{I}-\Delta, \quad B=\nu \Delta-i d \Delta^{2}$, where $\Delta$ is the Laplace operator. The operator $A \in \mathcal{L}(\mathfrak{X} ; \mathfrak{Y})$, and the operator $B \in \mathcal{C l}(\mathfrak{X} ; \mathfrak{Y})$ with $\operatorname{dom} B=\left\{x \in H^{4}(\Omega): \Delta x(s, t)=\right.$ $x(s, t)=0, s \in \partial \Omega\}$.

Lemma 7. [16] For any $\nu, \lambda, d \in \mathbb{R}$ the operator $B$ is strongly $(A, 0)$-radial.

The $A$-spectrum of operator $B$ consists of all points of the form

$$
\sigma^{A}(B)=\left\{\mu_{k} \in \mathbf{C}: \mu_{k}=\frac{\nu \lambda_{k}-i d \lambda_{k}^{2}}{\lambda-\lambda_{k}}, \text { if } k: \lambda_{k} \neq \lambda\right\}
$$

where $\lambda_{k}, \varphi_{k}$ are the same as in the Barenblatt-Zheltov-Kochina model. Thus $\mathfrak{Y}^{0}=\operatorname{span}\left\{\varphi_{k}: \lambda_{k}=\lambda\right\}, \mathfrak{Y}^{1}=\overline{\operatorname{span}}\left\{\varphi_{k}: \lambda_{k} \neq \lambda\right\}$. If the $A$-spectrum of operator $B$ is represented in the form of two components, which do not intersect and condition $(B)$ is fulfilled, then (13) takes the form

$$
\lim _{t \rightarrow 0+} \sum_{\mu_{k} \in \sigma_{i n}^{A}(B)}\left\langle\left(x(t)-x_{0}\right), \varphi_{k}\right\rangle \varphi_{k}=0, \sum_{\mu_{k} \in \sigma_{\text {fin }}^{A}(B)}\left\langle\left(x(\tau)-x_{\tau}\right), \varphi_{k}\right\rangle \varphi_{k}=0,
$$

where $\langle\cdot, \cdot\rangle$ is an inner product in $L_{2}(\Omega)$.

Let us proceed to the research of the optimal control problem (7), (9), (14), (37). Define $\mathfrak{U}=L_{2}(\Omega)$ and the operators $C=\mathbb{I}$. Take a closed convex set $H^{1}(\mathfrak{U})$. From Theorem 22 and Lemma 7 we have the following result.

Theorem 23. [16] Let the conditions of Lemma 7 be satisfied. Then for any $\tau \in \mathbb{R}_{+}$, $x_{0}, x_{\tau} \in \mathfrak{X}$ there exists a unique optimal control $u \in \mathfrak{U}_{a d}$ of solutions to the initial-final problem (37) for the model (7), (9), minimizing functional (20).

\section{Conclusion}

The article presents a review of results devoted to optimal control problems for linear Sobolev type equations with the Cauchy (Showalter-Sidorov) initial conditions or the initial-final conditions obtained by scientific school headed by Professor G.A. Sviridyuk. Abstract results in relatively bounded, sectorial and radial cases, allowing to study a wide class of non-classical models of mathematical physics are presented. Note that these results and methods have found applications in solving various problems, in particular, in the study of degenerate balance models (Leontief type models), as well as in finding the optimal dynamic measurements [34-36]. In addition, these results formed the basis of the study of optimal control problems for the non-autonomous linear Sobolev type equations [17], including the multipoint initial-final conditions [37-39], and were developed to the semi-linear case [9]. At present, there is an active extension of objects and methods of Sobolev type equations theory. Abstract results are transferred to the case of quasiBanach spaces, stochastic Sobolev type equations [35, 36, 40,41]. The study of positive solutions has started. 
Acknowledgment. The authors express their sincere gratitude to Professor G.A. Sviridyuk for useful consultations, constructive criticism and opportunities provided.

\section{References}

1. Barenblatt G.I., Zheltov Yu.P., Kochina I.N. Basic Concepts in the Theory of Seepage of Homogeneous Fluids in Fissurized Rocks. Journal of Applied Mathematics and Mechanics, 1960, vol. 24, no. 4, pp. 1268-1303. DOI: 10.1016/0021-8928(60)90107-6

2. Sviridyuk G.A., Efremov A.A. Optimal Control Problem for One Class of Linear Sobolev Type Equations. Russian Mathematics, 1996, vol. 40, no. 12, pp. 60-71.

3. Sviridyuk G.A., Fedorov V.E. Linear Sobolev Type Equations and Degenerate Semigroups of Operators. Utrecht; Boston; Köln; Tokyo, VSP, 2003. DOI: 10.1515/9783110915501

4. Zagrebina S.A. The Initial-Finite Problems for Nonclassical Models of Mathematical Physics. Bulletin of the South Ural State University. Series: Mathematical Modelling, Programming and Computer Software, 2013, vol. 6, no. 2, pp. 5-24. (in Russian)

5. Hoff N.J. Creep Buckling. Journal of the Aeronautical Sciences, 1956, no. 7, pp. 1-20. DOI: $10.1017 /$ S0001925900010106

6. Sviridyuk G.A., Shemetova V.V. Hoff Equations on Graphs. Differential Equations, 2006, vol. 42, no. 1, pp. 139-145. DOI: 10.1134/S0012266106010125

7. Sviridyuk G.A., Kazak V.O. The Phase Space of an Initial-Boundary Value Problem for the Hoff Equation. Mathematical Notes, 2002, vol. 71, no. 1-2, pp. 262-266. DOI: $10.4213 / \mathrm{mzm} 347$

8. Manakova N.A., Dyl'kov A.G. Optimal Control of the Solutions of the Initial-Finish Problem for the Linear Hoff Model. Mathematical Notes, 2013, vol. 94, no. 2, pp. 220-230. DOI: $10.1134 /$ S0001434613070225

9. Sviridyuk G.A., Manakova N.A. An Optimal Control Problem for the Hoff Equation. Journal of Applied and Industrial Mathematics, 2007, no. 2, pp. 247-253. DOI: $10.1134 /$ S1990478907020147

10. Zamyshlyaeva A.A., Tsyplenkova O.N. Optimal Control of Solutions of the ShowalterSidorov-Dirichlet Problem for the Boussinesq-Love Equation. Differential Equations, 2013, vol. 49, no. 11, pp. 1356-1365. DOI: 10.1134/S0012266113110049

11. Zamyshlyaeva A.A., Bychkov E.V., Tsyplenkova O.N. Mathematical Models Based on Boussinesq-Love Equation. Applied Mathematical Sciences, 2014, vol. 8, pp. 5477-5483. DOI: $10.12988 /$ ams.2014.47546

12. Dzektser E.S. [The Generalization of the Equations of Motion of Groundwater]. Dokl. Akad. Nauk SSSR, 1972, no. 5, pp. 1031-1033. (in Russian)

13. Sviridyuk G.A., Efremov A.A. Optimal Control of Sobolev Type Linear Equations with Relativity p-Sectorial Operators. Differential Equations, 1995, vol. 31, no. 11, pp. 1882-1890.

14. Manakova N.A., Dyl'kov A.G. Optimal Control of Solutions of the Initial-Finish Value Problem for a Evolutionary Models. Yakutian Mathematical Journal, 2012, vol. 19, no. 2, pp. 111-127. (in Russian)

15. Chen P.J., Gurtin M.E. On a Theory of Heat Conduction Involving Two Temperatures. Zeitschrift für angewandte mathematik und physik, 1968, vol. 19, pp. 614-627. DOI: $10.1007 / \mathrm{BF} 01594969$

16. Manakova N.A., Sviridyuk G.A. An Optimal Control of the Solutions of the InitialFinal Problem for Linear Sobolev Type Equations with Strongly Relatively p-Radial Operator. Springer Proceedings in Mathematics and Statistics, 2015, vol. 13, pp. 213-224. DOI: $10.1007 / 978-3-319-12145-1 \_13$ 
17. Sagadeeva M.A., Zagrebina S.A., Manakova N.A. Optimal Control of Solutions of a Multipoint Initial-Final Problem for Non-Autonomous Evolutionary Sobolev Type Equation. Evolution Equations and Control Theory, 2019, vol. 8, no. 3, pp. 473-488. DOI: 10.3934 /eect.2019023

18. Demidenko G.V., Uspenskii S.V. Partial Differential Equations and Systems Not Solvable with Respect to the Highest-Order Derivative. N.Y., Basel, Hong Kong, Marcel Dekker, 2003. DOI: $10.1201 / 9780203911433$

19. Favini A., Yagi A. Degenerate Differential Equations in Banach Spaces. N.Y., Basel, Hong Kong, Marcel Dekker, 1999. DOI: 10.1201/9781482276022

20. Sviridyuk G.A. [The Manifold of Solutions of an Operator Singular Pseudoparabolic Equation]. Dokldy Akademii Nauk SSSR, 1986, vol. 289, no. 6, pp. 1315-1318. (in Russian)

21. Al'shin A.B., Korpusov M.O., Sveshnikov A.G. Blow-up in Nonlinear Sobolev Type Equations. De Gruyter, 2011. DOI: 10.1515/9783110255294

22. Showalter R.E. The Sobolev Equation. Applicable Analysis, 1975, vol. 5, no. 1, pp. 15-22; vol. 5, no. 2, pp. 81-89. DOI: 10.1080/00036817508839103

23. Sviridyuk G.A., Zagrebina S.A. The Showalter-Sidorov Problem as a Phenomena of the Sobolev-Type Equations. The Bulletin of Irkutsk State University. Series: Mathematics, 2010, vol. 3, no. 1, pp. 104-125. (in Russian)

24. Keller A.V., Zagrebina S.A. Some Generalizations of the Showalter-Sidorov Problem for Sobolev-Type Models. Bulletin of the South Ural State University. Series: Mathematical Modelling, Programming and Computer Software, 2015, vol. 8, no. 2, pp. 5-23. (in Russian) DOI: $10.14529 / \mathrm{mmp} 150201$

25. Sviridyuk G.A., Zagrebina S.A. Verigin's Problem for Linear Equations of the Sobolev Type with Relatively p-Sectorial Operators. Differential Equations, 2002, vol. 38, no. 12, pp. 1745-1752. DOI: 10.1023/A:1023812213901

26. Sviridyuk G.A., Efremov A.A. Optimal Control for a Class of Degenerate Linear Equations. Doklady Akademii Nauk, 1999, vol. 364, no. 3, pp. 323-325.

27. Fedorov V.E., Plekhanova M.V. Optimal Control of Sobolev Type Linear Equations. Differential Equations, 2004, vol. 40, no. 11, pp. 1627-1637. DOI: 10.1007/s10625-004-0013-1

28. Fedorov V.E., Ruzakova O.A. Controllability in Dimensions One and Two of Sobolev-Type Equations in Banach Spaces. Mathematical Notes, 2003, vol. 74, no. 3-4, pp. 583-592. DOI: $10.1023 / \mathrm{A}: 1026160314352$

29. Zamyshlyaeva A.A. The Higher-Order Sobolev-Type Models. Bulletin of the South Ural State University. Series: Mathematical Modelling, Programming and Computer Software, 2014, vol. 7, no. 2, pp. 5-28. (in Russian)

30. Zamyshlyaeva A.A., Tsyplenkova O.N. Optimal Control of Solutions to Cauchy Problem for Sobolev Type Equation of Higher Order. Journal of Computational and Engineering Mathematics, 2014, vol. 1, no. 2, pp. 62-67.

31. Zamyshlyaeva A.A., Tsyplenkova O.N., Bychkov E.V. Optimal Control of Solutions to the Initial-Final Problem for Sobolev Type Equation of Higher Order. Journal of Computational and Engineering Mathematics, 2016, vol. 3, no. 2, pp. 57-67. DOI: 10.14529/jcem1602007

32. Bayazitova A.A. The Sturm-Liouville Problem on Geometric Graph. Bulletin of the South Ural State University. Series: Mathematical Modelling, Programming and Computer Software, 2010, no. 16 (192), issue 5, pp. 4-10. (in Russian)

33. Fedorov V.E [About Some Relations in the Theory of Degenerate Operator Semigroups]. Bulletin of the South Ural State University. Series: Mathematical Modelling, Programming and Computer Software, 2008, no. 15 (115), issue 7, pp. 89-99. (in Russian) 
34. Shestakov A.L., Keller A.V., Nazarova E.I. Numerical Solution of the Optimal Measurement Problem. Automation and Remote Control, 2012, vol. 73, no. 1, pp. 97-104. DOI: $10.1134 /$ S0005117912010079

35. Keller A.V., Shestakov A.L., Sviridyuk G.A., Khudyakov Y.V. The Numerical Algorithms for the Measurement of the Deterministic and Stochastic Signals. Springer Proceedings in Mathematics and Statistics, 2015, vol. 113, pp. 183-195. DOI: 10.1007/978-3-319-12145-1_11

36. Shestakov A.L., Sagadeeva M.A. Stochastic Leontieff-Type Equations with Multiplicative Effect in Spaces of Complex-Valued Noises. Bulletin of the South Ural State University. Series: Mathematical Modelling, Programming and Computer Software, 2014, vol. 7, no. 4, pp. 132-139. DOI: $10.14529 / \mathrm{mmp} 140412$

37. Zagrebina S.A., Soldatova E.A., Sviridyuk G.A. The Stochastic Linear Oskolkov Model of the Oil Transportation by the Pipeline. Springer Proceedings in Mathematics and Statistics, 2015, vol. 113, pp. 317-325. DOI: 10.1007/978-3-319-12145-1_20

38. Sviridyuk G.A., Zagrebina S.A., Konkina A.S. The Oskolkov Equations on the Geometric Graphs as a Mathematical Model of the Traffic Flow. Bulletin of the South Ural State University. Series: Mathematical Modelling, Programming and Computer Software, 2015, vol. 8, no. 3, pp. 148-154. DOI: 10.14529/mmp1503010

39. Zagrebina S.A., Konkina A.S. The Multipoint Initial-Final Value Condition for the NavierStokes Linear Model. Bulletin of the South Ural State University. Series: Mathematical Modelling, Programming and Computer Software, 2015, vol. 8, no. 1, pp. 132-136. DOI: $10.14529 / \mathrm{mmp} 150111$

40. Favini A., Sviridyuk G.A., Sagadeeva M.A. Linear Sobolev Type Equations with Relatively p-Radial Operators in Space of "Noises". Mediterranean Journal of Mathematics, 2016, vol. 13, no. 6, pp. 4607-4621. DOI: 10.1007/s00009-016-0765-x

41. Favini A., Zagrebina S.A., Sviridyuk G.A. Multipoint Initial-Final Value Problems for Dynamical Sobolev-Type Equations in the Space of "Noises". Electronic Journal of Differential Equations, 2018, vol. 2018, no. 128, pp. 1-10.

Received December 11, 2019

УДК 517.9

DOI: $10.14529 / \mathrm{mmp200101}$

\title{
ОПТИМАЛЬНОЕ УПРАВЛЕНИЕ В ЛИНЕЙНЫХ МАТЕМАТИЧЕСКИХ МОДЕЛЯХ СОБОЛЕВСКОГО ТИПА
}

\author{
А.А. Замышляева ${ }^{1}$, Н.А. Манакова ${ }^{1}$, О.Н. Цыпленкова \\ ${ }^{1}$ Южно-Уральский государственный университет, г. Челябинск, \\ Российская Федерация
}

В статье представлен обзор работ челябинской математической школы по уравнениям соболевского типа при исследовании задачи оптимального управления для линейных моделей соболевского типа с начальным условием Коши (Шоуолтера - Сидорова) 
или начально-конечным условием. Для выявления непустоты множества допустимых решений задачи управления используется уже хорошо зарекомендовавший себя при решении уравнений соболевского типа метод фазового пространства, заключающийся в редукции сингулярного уравнения к регулярному, определенному на некотором подпространстве исходного пространства и применении теории вырожденных (полу)групп операторов на случай относительно ограниченных, секториальных и радиальных операторов. В работе проводится редукция математических моделей к начальным (начально-конечным) задачам для абстрактного уравнения соболевского типа. Абстрактные результаты применены к исследованию задач управления для математической модели Баренблатта - Желтова - Кочиной, которая моделирует фильтрацию жидкости в трещинновато-пористой среде, модели Хоффа на графе, моделирующей динамику выпучивания двутавровых балок в конструкции, а также модели Буссинеска - Лява, описывающей продольные колебания в тонком упругом стержне с учетом инерции и при внешней нагрузке, либо распространения волн на мелкой воде.

Ключевые слова: уравнения соболевского типа; сильные решения; оптимальное управление; фазовое пространство; модель Баренблатта - Желтова - Кочиной; модель Хоффа; модель Буссинеска - Лява; модель Девиса; модель Чена - Гетина.

Алена Александровна Замышляева, доктор физико-математических наук, профессор, кафедра «Прикладная математика и программирование», ЮжноУральский государственный университет (г. Челябинск, Российская Федерация), zamyshliaevaaa@susu.ru.

Наталья Александровна Манакова, доктор физико-математических наук, доцент, кафедра «Уравнения математической физики», Южно-Уральский государственный университет (г. Челябинск, Российская Федерация), manakovana@susu.ru.

Ольга Николаевна Цыпленкова, кандидат физико-математических наук, кафедра «Уравнения математической физики», Южно-Уральский государственный университет (г. Челябинск, Российская Федерация), tcyplenkovaon@susu.ru.

Поступила в редакцию 11 декабря 2019 г. 